\title{
IT-Supported request management for clinical radiology: Analyzing Requirements through Contextual Interviews
}

\author{
Philipp Krop* \\ Samantha Straka* \\ philipp.krop@uni-wuerzburg.de \\ samantha.straka@uni-wuerzburg.de \\ HCI Group, University of Würzburg \\ Würzburg, Bavaria, Germany \\ Maximilian Ertl \\ Service Center Medical Informatics, University Hospital of \\ Würzburg \\ Würzburg, Bavaria, Germany \\ Ertl_M@ukw.de
}

\author{
Melanie Ullrich \\ Service Center Medical Informatics, University Hospital of \\ Würzburg \\ Würzburg, Bavaria, Germany \\ Ullrich_M@ukw.de \\ Marc Erich Latoschik \\ HCI Group, University of Würzburg \\ Würzburg, Bavaria, Germany \\ marc.latoschik@uni-wuerzburg.de
}

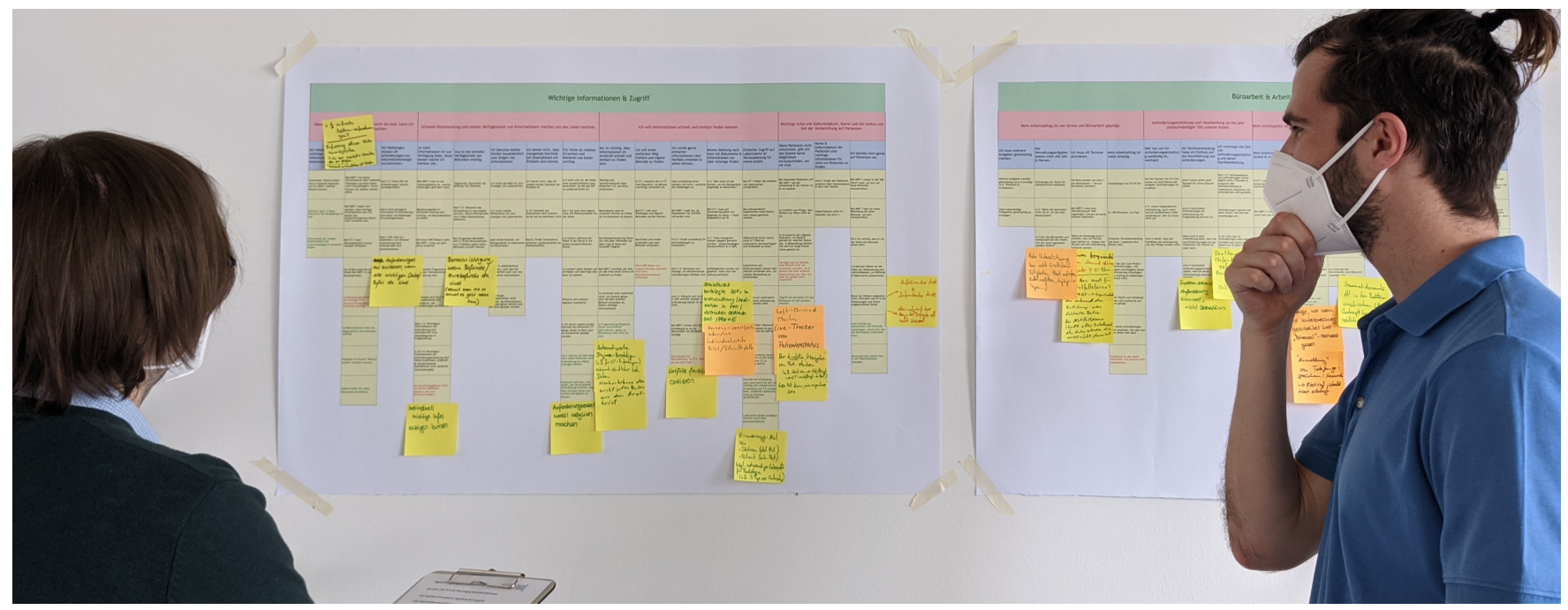

Figure 1: A mock-up of two physicians examining an affinity diagram, a large body of ordered observation data.

\begin{abstract}
Requests for radiological examinations in large medical facilities are a distributed and complex process with potential health-related risks for patients. A user-centered qualitative analysis with contextual interviews uncovered nine core problems, which hinder work efficiency and patient care: (1) Difficulties to access patient data \& requests, (2) the large number of phone calls, (3) restricted \& abused access rights, (4) request status difficult to track, (5) paper notes used
\end{abstract}

*Both authors contributed equally to this research.

This work is licensed under a Creative Commons Attribution International 4.0 License.

MuC '21, September 5-8, 2021, Ingolstadt, Germany

(c) 2021 Copyright held by the owner/author(s).

ACM ISBN 978-1-4503-8645-6/21/09.

https://doi.org/10.1145/3473856.3473992 for patient data, (6) lack of assistance for data entry, (7) frustration through documentation, (8) IT-systems not self-explanatory, and (9) conflict between physicians and radiologists. Contextual interviews were found to be a well fitting method to analyze and understand this complex process with multiple user roles. This analysis showed that there is room for improvement in the underlying IT systems, workflows and infrastructure. Our data gave useful insight into solutions to these problems and how we can use technology to improve all aspects of the request management. We are currently addressing those issues with a user-centered design process to design and implement a mobile application, which we will present in future work.

\section{CCS CONCEPTS}

- Human-centered computing $\rightarrow$ Field studies; User centered design; Contextual design; • Social and professional topics $\rightarrow$ Health information exchanges. 


\section{KEYWORDS}

Contextual Interviews, HCI, User-Centered Design, Affinity Diagram, Radiology

\section{ACM Reference Format:}

Philipp Krop, Samantha Straka, Melanie Ullrich, Maximilian Ertl, and Marc Erich Latoschik. 2021. IT-Supported request management for clinical radiology: Analyzing Requirements through Contextual Interviews. In Mensch und Computer 2021 (MuC '21), September 5-8, 2021, Ingolstadt, Germany. ACM, New York, NY, USA, 5 pages. https://doi.org/10.1145/3473856.3473992

\section{INTRODUCTION}

Radiological examinations such as X-rays or magnetic resonance tomography are common in modern hospitals. In our German partner hospital, a total number of 97000 examinations were requested and completed in 2017. As these examinations expose patients to high dosages of radiation, they should only be used when necessary. However, an internal analysis between 2011 and 2012 showed that doctors tend to request the full range of radiological examinations, even if only one specific procedure would be necessary. The dosage of radiation in these examinations is often underestimated by doctors $[3,4]$. As high dosages of radiation are harmful to the human body, this needs to be investigated. This work is part of an multidisciplinary project involving medical professionals, the hospitals' IT department, and computer science researchers. It strives to improve the radiological request management through increased digitization, machine learning, data mining, and user-centered design. As a first step to investigate weak points in the existing workflows, and chances for digitization, we chose to conduct a qualitative analysis with all participants involved.

Contribution: We report the results of a preliminary qualitative analysis that focuses on all users participating in the management of radiological examinations. We describe systems and workflows that are currently in use, and how they fail to support user needs. Our results indicate that there is much room for improvement not only with the IT systems but also with existing workflows and communication. At the end, we outline approaches on how we can use technology to improve radiological workflows in hospitals. In future work, we will present a mobile application addressing the identified problems which we are currently developing using a user-centered design process.

\section{RELATED WORK}

Technology has great potential in safety-critical environments like hospitals, where it can automate workflows and optimize communication and examinations. However, digitization in German hospitals lacks behind severely [17]. The Electronic Medical Record Adoption Model (EMRAM) [1] is a metric used worldwide to measure the extent of hospital digitization. A hospital with a score of zero has little to no digital workflows, while the workflows of a hospital with a score of seven are completely digital and use no paper. German hospitals achieve, on average, a score of 2.3, which means that their workflows are ranked well below the European average of 3.6. Hence, oftentimes workflows and patient documentation still rely on paper. This results in increased and redundant work for hospital workers. $44 \%$ of a medical doctor's working time was found to be spent on documentation instead of patient care [8]. A survey among the practitioners in the German medical doctors' union found that $80 \%$ of them think that an increase in digitization would improve their work [14]. However, this survey also indicated that $62 \%$ of hospitals offer no training for software. Because bad usability and user resistance are common reasons IT solutions in hospitals fail $[10,19]$, and only $11 \%$ of users think that the used software is user friendly, this shows that digitization alone is not enough.

\section{METHODS}

To understand the management of radiological examinations in a hospital context, we chose to follow a user-centered approach, which has been used to identify problems and solutions in hospitals with great success $[12,15,16,18]$. We conducted Contextual Interviews [9], which are a type of unstructured interviews, in which users and their everyday work are observed in the field. During those one-on-one interviews, we took the role of an active observer and shadowed the interviewee for two hours. While the interviewee was working as usual, we documented important information such as constraints, possibilities, user goals, opinions and emotions in a written protocol. Important observations were discussed as they happened. If possible, we took photos of important devices, documents or working environments. At the end of the interview, we clarified open questions with the interviewee. After concluding an interview, we met with stakeholders to interpret the written protocol. Important points were written on sticky notes from the user's perspective. We aggregated these notes into a hierarchically ordered affinity diagram. The diagram was expanded with additional data after each subsequent interview. The wall walking method as proposed by Holtzblatt et al. [9] was then used in a joint meeting with all roles participating in the process to find and discuss the most important problem areas and solutions. This is shown in Figure 1.

We followed this workflow as close as possible, but adjusted it to properly work in the hospital environment, which is illustrated in Figure 2. Due to privacy concerns, we were not allowed to take photos of interfaces, workspaces and important documents. Thus, we had to draw each work environment, screen, or document by hand. The project was endorsed by the management. Several available doctors took part as much as their time constraints allowed. Resulting data gaps were filled by conducting a focus group [13]. Before the analysis we digitized the affinity diagram for better readability. After the analysis of the affinity diagram, we joined the weekly meeting between the senior doctors to confirm and expand our results with more multidisciplinary participants.

\subsection{Participants}

We interviewed twenty-one participants (12 female, nine male, $\left.M_{\text {age }}=30.78, S D_{\text {age }}=6.78\right)$. This involved eleven doctors that issue radiological requests, four assistants in the radiology station who set the examination date, three radiologists who plan the requested examination in detail and document the results, and four student interns in their practical year. During the analysis, we documented the respective user group on each note to identify the needs and wishes of each user group. Five physicians (two female, three male, $\left.M_{\text {age }}=38.75, S D_{\text {age }}=9.68\right)$ participated in 


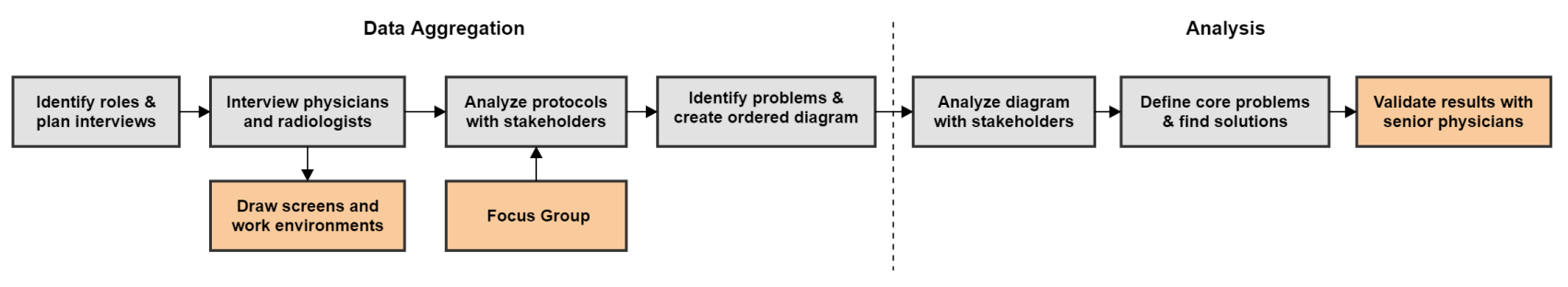

Figure 2: We chose to follow a user-centered design approach by conducting contextual interviews. Multiple changes were needed to properly adapt the method to the hospital environment and to gather further information. These changes are highlighted in orange.

the additional focus group, with each participant having different responsibilities in the request management.

\section{RESULTS}

We worked with the end-users and the hospitals IT department for every possible step of the contextual interviews. This approach was well received, as many users told us they felt heard for the first time. We found nine problems that interact with and exacerbate each other. We determined their severity of each problem area as either Low, Medium or High. A problem with high severity has a major negative influence on the request management and should be addressed as soon as possible. Medium problems are still hindering current workflows, while low ranked problems could be fixed through improving the process in general. Our results, their severity, and potential solutions are summarized in table 1.

(1) Difficulties to access patient data \& requests. Patient data is difficult to find using existing systems, and cannot be efficiently shared with colleagues. Laboratory results, risk factors, and the results of radiological examinations are distributed across systems, are perceived as hard to find and require multiple clicks to open. Thus, we propose that information is aggregated in easy to find places. For example, all patient data should be accessible whenever a user clicks on a patient, with the most important information shown in a central place. The results of every previous examination and laboratory results should be listed there in chronological order. Further, patient data and request management is only available on few computers per station, but not during an examination. It requires physicians to either prepare paper notes or pause an examination whenever they need patient data or have to create requests. This could be solved by making patient data and request management available on mobile devices. Mobile access to information was identified as a key factor to implement successful request management [11], and can greatly improve workflows in hospital settings [2].

(2) Large number of phone calls. With every request, medical doctors have to call the radiology station to rule out unnecessary procedures and set the date for the examination. This is a major cause of frustration, interrupts and efficiency problems, as has previously been shown by [5]. As a call has to be made for every request, most of these calls end up in a queue, where doctors spend twenty to thirty minutes. Since calls were perceived as the fastest way to access patient data and the results of an examination, physicians often called the radiology station just for updates. To make matters worse, some doctors try to circumvent the queue by calling radiologists directly. Radiologists are then interrupted during screenings, which in turn has been shown to increase errors [21]. A solution would be to provide asynchronous communications channels [5], e.g. chats, as answering them can be postponed, unlike calls. Automatic scheduling would eliminate the need to call the radiology station to set an examination date. There were some tasks where calls were the preferred method of communication, like emergencies, where quick access and coordination from both sides is necessary. We expect our solutions to rule out unnecessary phone calls and shorten the queue for emergencies.

(3) Restricted \& abused access rights. Medical assistants or student interns have no access rights to patient data and request management. This not only hinders them in their work, but also prevents them from learning how to create well-written requests. In theory, these access rights are in place to protect privacy. In practice however, physicians give away their login data to assistants and student interns. Neither of them liked this workaround, but felt it was needed to perform their daily work. Using the access of higher-ups is a serious problem, as responsibility can not be tracked. We propose that student interns and assistants gain restricted access rights to patient data and request management. To ensure quality, requests created by student interns should be checked by higher-ups before being send to the radiology.

(4) Request status difficult to track. Both physicians and radiologists stated that it is difficult to track the status of an examination. Even though physicians can see the current status, not every step along the workflow has its own status. For some of these steps, the quickest way to get the status is to call the radiology station. They wished to see this status being both more detailed and more frequently updated. Because of this, physicians often did not notice when the results of an examination were available. Thus, we propose both a timeline, where the current status can be seen at a glance, as well as a detailed protocol for better comprehensibility. For every step, a timestamp and the responsible person should be visible. Physicians should be notified whenever the results of an examination are available. 


\begin{tabular}{|c|c|c|}
\hline Problem area & Severity & Potential Solutions \\
\hline (1) Difficulties to access patient data \& requests & High & $\begin{array}{l}\text { 1. Aggregate information in easy to find places. } \\
\text { 2. Provide mobile requests \& patient data. }\end{array}$ \\
\hline (2) Large number of phone calls & High & $\begin{array}{l}\text { 1. Support asynchronous communication channels. } \\
\text { 2. Automate examination scheduling. }\end{array}$ \\
\hline (3) Restricted \& abused access rights & High & $\begin{array}{l}\text { 1. Provide restricted access to student interns. } \\
\text { 2. Let student interns create supervised requests. }\end{array}$ \\
\hline (4) Request status difficult to track & High & $\begin{array}{l}\text { 1. Show current request status. } \\
\text { 2. Notify users about important status changes. }\end{array}$ \\
\hline (5) Paper notes used for patient data & Medium & $\begin{array}{l}\text { 1. Provide mobile requests \& patient data. } \\
\text { 2. Use speech to create requests on the go. }\end{array}$ \\
\hline (6) Lack of assistance for data entry & Medium & $\begin{array}{l}\text { 1. Add designated fields for specific information. } \\
\text { 2. Auto-complete requests with patient data. }\end{array}$ \\
\hline (7) Frustration through documentation & Medium & $\begin{array}{l}\text { 1. Provide mobile requests \& patient data. } \\
\text { 2. Use speech to create requests on the go. }\end{array}$ \\
\hline (8) IT-Systems not self-explanatory & Low & $\begin{array}{l}\text { 1. Add explanatory symbols \& tool-tips. } \\
\text { 2. Design interfaces with usability in mind. }\end{array}$ \\
\hline (9) Conflict between physicians and radiologists & Low & $\begin{array}{l}\text { 1. Support asynchronous communication channels. } \\
\text { 2. Allow changes rather than sending new requests. }\end{array}$ \\
\hline
\end{tabular}

Table 1: The problem areas with their severity and possible solutions.

(5) Paper notes used for patient data. As patient data can only be accessed though few computers in the station, physicians create paper lists and use them for mobile access. We observed them taking notes on paper during ward rounds, which they had to enter into the system later, with sometimes hours between talking to the patient and issuing the request. This is error-prone and time-intensive. The users are aware of the disadvantages paper lists bring, but choose to use them anyway. This also could be solved by making patient data and request management available on mobile devices. Allowing physicians to issue and manage requests during the ward round would decrease the time between examination and request creation, thus preventing information loss and errors that occur when using analogue materials. Even though smartphones were given to physicians, they doubted that phone keyboards would be suitable for entering often large blocks of patient data. For this, speech recognition might be a suitable interaction paradigm.

(6) Lack of assistance for data entry. When needing multiple examinations at once, a physician would often send one specific request, but add the others in the comment-section of the request form. This was done because the request form only allows a single examination, as required by accounting reasons. The comment field was also used for important information for which there was no designated field in the request form, such as previous results or specific allergies. To prevent physicians from writing important information into the comment section, designated fields should be provided. Besides that, known patient data like the gender or allergies had to be manually added to each request, which was perceived as redundant work. We suggest that known patient data is added automatically to designated fields.

(7) Frustration through documentation. Physicians have to balance patient care with supervision of trainees and office work. Every role we interviewed was frustrated about the time spent for request management and documentation instead of patient care. Mobile access to patient data and request management will allow physicians to spend less time in the office and more with the patients. Also, as documentation often consists of large text blocks, speech recognition and readily available templates would lead to faster data entry.

(8) IT-Systems not self-explanatory. Previous evaluations of hospital systems found that IT-systems are lacking in usability, especially regarding individualization, self-descriptiveness, learnability, and the suitability for specific tasks [7]. Similar, we found that users were often puzzled about the meaning of specific symbols \& icons, and why the system performed certain actions - usually actions they did not intent. When asked about certain buttons on their screen, they often had no idea about their functionality, and could not easily find out what they are supposed to do. This could easily be solved by redesigning the interfaces according to usability guidelines. Self-explanatory symbols \& tool-tips could be used to help users better understand the system's functionality.

(9) Conflict between physicians and radiologists. We observed a mutual feeling of adversary between physicians and radiologists: physicians did not want to spend more time than necessary on request management. Thus, they copy and paste patient data in the form of large text blocks, which in turn leads to radiologists needing to spend more time than necessary finding the important parts in the text. This data is often unspecified, which leads to requests lacking in quality. As requests can not be changed, radiologists have to send them back and physicians need to create a new, improved request or call the radiologists to clarify open questions. This sometimes doubles the work for a single examination on both sides. Both, radiologists and physicians, blame the other side for wasting their time. We believe that providing asynchronous communication channels like chats, and being able to change requests, will balance 
the workload between physicians and radiologists, improving their adversary relationship.

\section{CONCLUSION}

All in all, users were not satisfied with the current status quo and workflows for request management. Many of our results are in line with research published up to twenty years ago for other health facilities [20]. However, it seems that reported problems are agnostic to the advances in IT development. Hence, our results indicate that it is necessary to properly analyze current states individually for similar tasks. The contextual interview method has shown to be a valid method to understand the complex and distributed workflows of the hospital. It gave us insight into how different roles work on their specific tasks. Problems and deficits in the underlying IT systems and workflows lead to sub-optimal patient care and dissatisfaction. Failed launches of IT-systems in hospitals are often blamed on the users unwillingness to learn and adapt to new systems [6]. However, we strongly believe that improving user interfaces and additions to the current workflows instead of complete replacements should help users adapt to new systems. Our data gives great insight into how these problems could be improved or solved, e.g. by providing mobile access to information, reducing phone calls, and implement user-friendly ways to manage requests.

Concurrent to our analysis, the partner hospital supplied each physician with an iPhone XR and started the development of a mobile app. We and the hospital's IT-department see this as a chance to implement our proposed solutions. Thus, we strive to improve request management by giving physicians mobile access to patient data, and to create requests on the go. The status of a request will be accessible through timelines and detailed protocols and changelogs. Communication should work asynchronously without requiring phone calls, e.g. through chats. Student interns and assistants will have restricted access to patient data and request management under supervision. Currently, we are using a user-centered design approach to prototype solutions and optimize usability and user experience, which we will present in future work.

\section{ACKNOWLEDGMENTS}

This research was funded by the Bavarian State Ministry for Science and Art within the "Digitalisierungszentrum für Präzisions- und Telemedizin" (DZ.PTM) project, as part of the master plan "BAYERN DIGITAL II".

\section{REFERENCES}

[1] HIMSS Analytics North America. 2021. Electronic Medical Record Adoption Model. https://www.himssanalytics.org/emram

[2] Elske Ammenwerth, Anke Buchauer, Bernd Bludau, and Reinhold Haux. 2000 Mobile information and communication tools in the hospital. International fournal of Medical Informatics 57, 1 (2000), 21-40. https://doi.org/10.1016/S13865056(99)00056-8

[3] Lars Borgen, Erling Stranden, and Ansgar Espeland. 2010. Clinicians' justification of imaging: do radiation issues play a role? Insights into imaging 1, 3 (2010), 193-200.

[4] Nicholas Brown and Lee Jones. 2013. Knowledge of medical imaging radiation dose and risk among doctors. Fournal of medical imaging and radiation oncology 57, 1 (2013), 8-14.

[5] Enrico Coiera and Vanessa Tombs. 1998. Communication behaviours in a hospital setting: an observational study. BMF (Clinical research ed.) 316, 7132 (feb 1998), 673-6. https://doi.org/10.1136/bmj.316.7132.673

[6] Alan F. Dowling. 1980. Do Hospital Staff Interfere with Computer System Implementation? , 302-317 pages. https://doi.org/10.1007/978-1-4613-8674-2_19
[7] K.-C. Hamborg, B. Vehse, and H.-B. Bludau. 2004. Questionnaire Based Usability Evaluation of Hospital Information Systems. Electronic fournal of Information Systems Evaluation 7, 1 (2004), 21-30.

[8] HIMSS Europe. 2015. Auf den Spuren der Zeitdiebe im Krankenhaus. https: //www.dragon-speaking.de/download/HIMSS-Europe-Studie.pdf

[9] Karen Holtzblatt, Jessamyn Burns Wendell, and Shelley Wood. 2004. Rapid contextual design: a how-to guide to key techniques for user-centered design. Elsevier, 500 Sansome Street, Suite 400, San Francisco, CA 94111.

[10] Ursula Hübner, Moritz Esdar, Jens Hüsers, Jan-David Liebe, Laura Naumann, Johannes Thye, and Jan-Patrick Weiß. 2020. IT-Report Gesundheitswesen 2020 Wie reif ist die Gesundheits-IT aus Anwender-Perspektive? Befragung ärztlicher und pflegerischer Krankenhaus-Direktoren *innen in Deutschland, Österreich und der Schweiz. Technical Report. Forschungsgruppe Informatik im Gesundheitswesen (IGW) - Hochschule Osnabrück.

[11] Fiona Lee, Jonathan M. Teich, Cynthia D. Spurr, and David W. Bates. 1996. Implementation of Physician Order Entry: User Satisfaction and Self-reported Usage Patterns. Emerging Infectious Diseases 3, 1 (1996), 42-55. https://doi.org/10.1136/ jamia.1996.96342648

[12] Fan-Yun Pai and Kai-I Huang. 2011. Applying the Technology Acceptance Model to the introduction of healthcare information systems. Technological Forecasting and Social Change 78, 4 (may 2011), 650-660. https://doi.org/10.1016/J. TECHFORE.2010.11.007

[13] Fatemeh Rabiee. 2004. Focus-group interview and data analysis. Proceedings of the nutrition society 63, 4 (2004), 655-660. https://doi.org/10.1079/PNS2004399

[14] T. Rudolf and S. Schmidt. 2016. Digitalisierung im Krankenhaus. Medizintechnik (Cologne) 136, 6 (2016), 28-30.

[15] Warner V. Slack and Howard L. Bleich. 1999. The CCC system in two teaching hospitals: A progress report. International fournal of Medical Informatics 54, 3 (jun 1999), 183-196. https://doi.org/10.1016/S1386-5056(99)00006-4

[16] John B Smelcer, Hal Miller-Jacobs, and Lyle Kantrovich. 2009. Usability of electronic medical records. Fournal of usability studies 4, 2 (2009), 70-84.

[17] Victor Stephani, Reinhard Busse, and Alexander Geissler. 2019. Benchmarking der Krankenhaus-IT: Deutschland im internationalen Vergleich. In KrankenhausReport 2019. Springer Berlin Heidelberg, Berlin, Heidelberg, 17-32. https://doi. org/10.1007/978-3-662-58225-1_2

[18] Karin A. Thursky and Michael Mahemoff. 2007. User-centered design techniques for a computerised antibiotic decision support system in an intensive care unit. International fournal of Medical Informatics 76, 10 (2007), 760-768. https://doi. org/10.1016/j.ijmedinf.2006.07.011

[19] Johannes Thye, Ursula Hübner, Moritz Esdar, Jens Hüsers, Jens Rauch, Jan-David Liebe, and Jan-Patrick Weiß. 2018. IT-Report Gesundheitswesen 2018 - Schwerpunkt - Wie reif ist die IT in deutschen Krankenhäusern? Technical Report. Forschungsgruppe Informatik im Gesundheitswesen (IGW) - Hochschule Osnabrück.

[20] A. Tonks and R. Smith. 1996. Doctors' use of electronic medical records systems in hospitals: cross sectional survey. British Medical fournal 313, 7055 (1996), 438. https://doi.org/10.1136/bmj.313.7055.438

[21] Johanna I Westbrook, Magdalena Z Raban, Scott R Walter, and Heather Douglas. 2018. Task errors by emergency physicians are associated with interruptions, multitasking, fatigue and working memory capacity: a prospective, direct observation study. BMF quality \& safety 27,8 (2018), 655-663. 